\title{
New Rural Cooperative Medical Service Quality Evaluation Method Considering Uncertainty Decision-making Information
}

\author{
FU Mengyan \\ XiDian University, School of Economics and Management, Xifeng Road, Xinglong 266, Xian, China
}

\begin{abstract}
New raral cooperative medical system is one of the strategy to construct new rural communities. In order to effectively improve the quality of the new rural medical services and address the existing medical problems, a hybrid multi-attribute evaluation method has been given, based on taking into account the uncertain of decision-making information and valuation indicator. Firstly, the conception of interval two-tuple linguistic has been explained. And then, this paper describe the problem of the new rural cooperative medical service quality evaluation and propose a TOPSIS evaluation method based on two-tuple linguistic. Finally, an example analysis shows the practicality and effectiveness of the proposed method.
\end{abstract}

Keywords: medical service; uncertain; interval two-tuple linguistic; New raral cooperative medical system; TOPSIS

\section{Introduction}

In order to solve the medical problems of "difficulty in seeing a doctor, high cost of getting a treatment, illness-caused poverty and poverty returned by illness", China central Committee clearly put forward the governments at all levels should actively guide farmers to establish a serious illness co-ordination of the new rural cooperative medical system The New Rural Cooperative Medical System (NRCMS) refers to the farmers' mutual medical assistance system, which is organized, guided and supported by the government, participated by farmer voluntarily.raising fund through multiple channels like individual, collective and government ${ }^{[1]}$. The New Rural Cooperative Medical System played a positive role not only in ensuring farmers access to basic health services and reducing the illness-related poverty, but also in promoting a harmonious society and new rural construction ${ }^{[2]}$. At present, the research on the new rural cooperative medical system mainly involves the construction of the new rural cooperative medical insurance system ${ }^{[3]}$, the financing of the new rural cooperative medical system ${ }^{[4]}$, the compensation of the new rural cooperative medical system ${ }^{[5]}$ and the new rural cooperative medical fund Segmentation ratio problem ${ }^{[6]}$ and so on.

Most of the existing researches have remained in the aspects of system and institution-building problems, but the performance evaluation of new rural cooperative medical service is less involved. The valuation of new rural cooperative medical service, which not only can help find a series of problems in its implementation process, and then promote the harmonious society and the new socialist countryside Construction, but also can improve the quality of rural medical service on the basis of solving these problems, has practical significance. Therefore, on the basis of the above analysis, in order to effectively solve the problems in the new rural cooperative medical service and effectively improve the quality of the new rural cooperative medical service, this paper proposes a new rural cooperative medical service Performance Evaluation Methods with uncertainty decision-making process and index.

\section{Preliminary Knowledge}

In decision-making, both of the decision-making problem itself and the evaluation process are complex and uncertain, and the decision-maker's understanding of the problem is limited and incomplete. In addition, the evaluation index related to the decision-making problem may also have uncertainty. In order to scientifically and rationally describe this uncertainty, many scholars use language evaluation information to evaluate. For purpose of dealing with the information of language evaluation effectively and reducing the loss and distortion of information in the process of information aggregation, this paper uses interval two-tuple semantics to aggregate the evaluation information for different types of language evaluation information in the evaluation of new rural cooperative medical service.

\subsection{The basic concepts of binary semantics}

The concept of binary semantics was first proposed by Herrera and Martinez ${ }^{[7]}$ in 2000, which is a two-tuple $\left({ }_{i}, \alpha\right)$ based on the notion of symbol conversion and is used to represent the language information given by the expert, In

the Language Evaluation Sets $S, S_{i}$ is an evaluation phrase, $\alpha$ is a symbolic transfer value, is used to represent the difference between the linguistic information and the closest linguistic phrase in the linguistic phrase set.

Definition1.1 $^{[8]}$ Let $s_{i} \in S$ bean evaluation phrase, the 


\section{International Journal of Science and Research (IJSR) \\ ISSN (Online): 2319-7064}

Index Copernicus Value (2015): 78.96 | Impact Factor (2015): 6.391

two-tuple linguistic form of $S_{i}$ can be obtained by the

formula (1).

$\delta: S \rightarrow S \times[-0.5,0.5), \delta\left(s_{i}\right)=\left(s_{i}, 0\right)$

Definition1.2 $2^{[8-9]}$ Let $S=\left\{s_{0}, s_{1}, \ldots, S_{g}\right\}$ be Language

Evaluation Sets, $\left(s_{i}, \alpha\right)$ is a two-tuple linguistic information.

There is a function to convert the binary semantics into numerical form, namely:

$$
\begin{aligned}
& \Delta^{-1}: S \times\left[-\frac{1}{2 g}, \frac{1}{2 g}\right) \rightarrow[0,1] \\
& \Delta^{-1}\left(s_{i}, \alpha\right)=\frac{i}{g}+\alpha=\beta
\end{aligned}
$$

\subsection{The basic concept of two-tiered semantics}

Interval two-tuple linguistic ${ }^{[10]}$ is proposed on the basis of two-tuple linguistic. In real life, because of the subjective and objective factors such as knowledge structure, time and experience, the evaluation value of the indicator given by the decision maker may be between the two language phrases, which deal with by traditional decision-making method will make some information distorted and lost. Therefore, in order to solve this kind of problem effectively, the interval two-tuple linguistic is described.

Definition2.1 $^{[10]}$ Let $S=\left\{s_{0}, s_{1}, \ldots, s_{g}\right\}$ be Language Evaluation Sets, $\left[\left(s_{k}, \alpha_{1}\right),\left(s_{l}, \alpha_{2}\right)\right]$ is an interval two-tuple linguistic information and $\left(s_{k}, \alpha_{1}\right) \leq\left(s_{l}, \alpha_{2}\right)$. The interval two-tuple linguistic information can be transformed into interval values like $\left[\beta_{1}, \beta_{2}\right]\left(\beta_{1}, \beta_{2} \in[0,1]\right)$ and $\Delta\left[\beta_{1}, \beta_{2}\right]=\left[\left(s_{k}, \alpha_{1}\right),\left(s_{l}, \alpha_{2}\right)\right]$ by the following formulas (4) and (5)。

$$
\left\{\begin{array}{l}
s_{k}, k=\operatorname{round}\left(\beta_{1}, g\right) \\
s_{l}, l=\operatorname{round}\left(\beta_{2}, g\right) \\
\alpha_{1}=\beta_{2}-\frac{k}{g}, \alpha_{1} \in\left[-\frac{1}{2 g}, \frac{1}{2 g}\right] \\
\alpha_{2}=\beta_{2}-\frac{l}{g}, \alpha_{2} \in\left[-\frac{1}{2 g}, \frac{1}{2 g}\right]
\end{array}\right.
$$

$$
\Delta^{-1}\left[\left(s_{k}, \alpha_{1}\right),\left(s_{l}, \alpha_{2}\right)\right]=\left[\frac{k}{g}+\alpha_{1}, \frac{l}{g}+\alpha_{2}\right]=\left[\beta_{1}, \beta_{2}\right]
$$

Definition2.2 ${ }^{[10]}$ Assume an interval two-tuple linguistic set like

$$
\tilde{X}=\left\{\begin{array}{l}
{\left[\left(r_{1}, \alpha_{1}\right),\left(t_{1}, \varepsilon_{1}\right)\right],\left[\left(r_{2}, \alpha_{2}\right),\left(t_{2}, \varepsilon_{2}\right)\right],} \\
\ldots,\left[\left(r_{n}, \alpha_{n}\right),\left(t_{n}, \varepsilon_{n}\right)\right]
\end{array}\right\},
$$

let $\tilde{w}=\left(\tilde{w}_{1}, \tilde{w}_{2}, \ldots, \tilde{w}_{n}\right)(j=1,2, \ldots, n)$ be the wight of all attributes, where $\tilde{w}_{j} \in[0,1], \quad \sum_{j=1}^{n} \tilde{w}_{j}=1$.Interval Two-tuple weighted Aggregation Operator(ITWA) can be defined by formula(6)

$$
\operatorname{ITWA}(X)=\Delta\left(\sum_{j=1}^{n} \tilde{w}_{j} \Delta^{-1}\left(r_{j}, \alpha_{j}\right), \sum_{j=1}^{n} \tilde{w}_{j} \Delta^{-1}\left(t_{j}, \varepsilon_{j}\right)\right)
$$

Definition2.3 ${ }^{[8-9]}$ For two an interval two-tuple linguistic information

$$
\tilde{a}=\left[\left(r_{1}, \alpha_{1}\right),\left(t_{1}, \varepsilon_{1}\right)\right]
$$

$\tilde{b}=\left[\left(r_{2}, \alpha_{2}\right),\left(t_{2}, \varepsilon_{2}\right)\right]$, the distance measure between $\tilde{a}$ and $\tilde{b}$,denoted as $D(\tilde{a}, \tilde{b})$, satisfy the following formula(7)

$$
D(\tilde{a}, \tilde{b})=\Delta\left[\frac{1}{2}\left(\begin{array}{l}
\left|\Delta^{-1}\left(r_{1}, \alpha_{1}\right)-\Delta^{-1}\left(r_{2}, \alpha_{2}\right)\right| \\
+\left|\Delta^{-1}\left(t_{1}, \varepsilon_{1}\right)-\Delta^{-1}\left(t_{2}, \varepsilon_{2}\right)\right|
\end{array}\right)\right]
$$

\section{Construct the Evaluation Model of the New Rural Cooperative Medical service}

In order to promote the harmonious society and the construction of new socialist countryside and solve the problems in traditional rural medical service, the CPC Central Committee put forward the concept of new rural cooperative medical care. New Rural Cooperative Medical System is the product in the process of building a new socialist countryside, take aim at improving the quality of new rural medical services, and promoting the construction of new rural areas. The purpose of this paper is to evaluate the quality of new rural cooperative medical service, and to explore and solve the problems in the implementation of the new rural cooperative medical system. Let $\mathrm{DH}_{h}$ $(h=1,2, \ldots, H)$ denote the $h$ th decision-maker; $A_{p}$ $(p=1,2, \ldots, P)$ denote the $p$ th new rural cooperative 


\section{International Journal of Science and Research (IJSR) \\ ISSN (Online): 2319-7064 \\ Index Copernicus Value (2015): 78.96 | Impact Factor (2015): 6.391}

medical institutions; $C_{q} q=1,2, \ldots, Q$ denote the $q$ th evaluation index; $C_{q_{\theta}}(\theta=1,2, \ldots, \Theta)$ denote the $p$ th secondary index under the $q$ th primary index; $\lambda_{h}$ $(h=1,2, \ldots, H)$ denote the weight of the $h$ th decision-maker, which satisfies $\sum_{h=1}^{H} \lambda_{h}=1 ; v_{q}$ denote the weight of the $q$ th evaluation index; $v_{q_{\theta}}$ denote the weight of the $p$ th secondary index under the $q$ th primary index, with $\sum_{\theta=1}^{\Theta} v_{q_{\theta}}=1 ; \quad D_{h}=\left[d_{p q_{\theta}}^{h}\right]_{P \times Q_{\Theta}}$ denotes the evaluation matrix, where $d_{p q_{\theta}}^{h}$ denote the $h$ th decision-maker evaluates the value of the $p$ th new rural cooperative medical institution based on the $q_{\theta}$ th index.In addition, decision makers may give their evaluation results according to different language phrase evaluation sets. In order to solve this problem effectively, the TOPSIS group decision-making method based on interval two-tuple semantic is presented as follows:

Step1: Construct new rural cooperative medical service evaluation index system. First of all, the relevant literature review [11-14], summarize the evaluation index system in the literature; then interview the relevant experts, and sort out the expert opinions; Finally, we modify the index system according to China's national conditions and relevant policies, and then, build up the evaluation index system of new rural cooperative medical service in our country, including infrastructure construction, system construction, team construction, network construction, medical cost, continuity and so on several aspects, each index is also refined into a number of sub indicators. This paper constructs the new rural cooperative medical service evaluation index system and the corresponding weight as shown in Table 1.

Table 1: Criteria For Evaluation

\begin{tabular}{|c|c|}
\hline Major criterion & Sub criterion \\
\hline \multirow{4}{*}{$\begin{array}{l}C_{1}: \text { Infrastructure } \\
\text { Construction }(0.18)\end{array}$} & $C_{11}:$ Advanced and precise facility 0.25 ) \\
\hline & $C_{12}:$ Hygienic conditions $(0.20)$ \\
\hline & $C_{13}:$ Convenience of traffic $(0.25)$ \\
\hline & $\begin{array}{l}C_{14}: \text { Convenient for medical treatment } \\
\text { process }(0.30)\end{array}$ \\
\hline \multirow{2}{*}{$\begin{array}{l}C_{2}: \text { System } \\
\text { Construction }(0.09)\end{array}$} & $\begin{array}{l}C_{21}: \text { completeness of medical system } \\
(0.35)\end{array}$ \\
\hline & $\begin{array}{l}C_{22} \text { : The ability of hospital to solve } \\
\text { problems in time }(0.35)\end{array}$ \\
\hline
\end{tabular}

\begin{tabular}{|c|c|}
\hline & $\begin{array}{l}\quad C_{23}: \text { The ability of the system to cope } \\
\text { with changes for the internal and external } \\
\text { environment }(0.30)\end{array}$ \\
\hline \multirow{4}{*}{$\begin{array}{l}C_{3}: \text { Team } \\
\text { Construction }(0.23)\end{array}$} & $\begin{array}{l}C_{31}: \text { Professional capability of medical } \\
\text { staff }(0.30)\end{array}$ \\
\hline & $C_{32}:$ Medical ethics $(0.20)$ \\
\hline & $C_{33}:$ Ability to cooperate with tea, $(0.25)$ \\
\hline & $C_{34}:$ Ability to information sharing $(0.25)$ \\
\hline \multirow[t]{2}{*}{$\begin{array}{l}C_{4} \text { : Network } \\
\text { Construction }(0.12)\end{array}$} & $\begin{array}{l}C_{41} \text { :The completion of : Hospital } \\
\text { network construction }(0.55)\end{array}$ \\
\hline & $\begin{array}{l}C_{42}: \text { The usability of the network for } \\
\text { both doctor and other people }(0.45)\end{array}$ \\
\hline \multirow{3}{*}{$\begin{array}{l}C_{5}: \text { Medical Costs } \\
(0.17)\end{array}$} & $\begin{array}{l}C_{51} \text { : Reasonability of basic medical } \\
\text { expenses }(0.35)\end{array}$ \\
\hline & $\begin{array}{l}C_{52}: \text { Reasonability of expert } \\
\text { consultation expenses }(0.35)\end{array}$ \\
\hline & $\begin{array}{l}C_{53}: \text { Reasonability of medicine } \\
\text { expenses }(0.30)\end{array}$ \\
\hline \multirow{2}{*}{$\begin{array}{l}C_{6}: \\
\text { Continuity }(0.21)\end{array}$} & $\begin{array}{l}C_{61}: \text { Ability to prevent the common } \\
\text { disease }(0.50)\end{array}$ \\
\hline & $\begin{array}{l}C_{62}: \text { Regular review and care of } \\
\text { critically ill patients }(0.50)\end{array}$ \\
\hline
\end{tabular}

Step2: Establish the language phrase evaluation matrix $D_{h}=\left[d_{p q_{\theta}}^{h}\right]_{P \times Q_{\Theta}}$, and then transform the language phrase evaluation matrix into the interval two-tuple linguistic evaluation matrix $\tilde{D}_{h}=\left[\tilde{d}_{p q_{\theta}}^{h}\right]_{P \times Q_{\Theta}}=\left[\left(\tilde{r}_{p q_{\theta}}^{h}, 0\right),\left(\tilde{t}_{p q_{\theta}}^{h}, 0\right)\right]_{P \times Q_{\Theta}}$. If the expert gives only one evaluation phrase, it will be converted into two-tuple linguistic with same upper and lower bounded; if the expert gives two linguistic phrases, the evaluation value between the two evaluation phrases.

Step3: Weigh the secondary index evaluation value to obtain the first grade index evaluation matrix as shown in formula (8)

$$
\begin{aligned}
& \tilde{r}_{p q}^{h}=\left[\left(\tilde{r}_{p q}^{h}, 0\right),\left(\tilde{t}_{p q}^{h}, 0\right)\right] \\
& =\Delta\left[\sum_{\theta=1}^{\Theta} v_{q_{\theta}} \Delta^{-1}\left(\tilde{r}_{p q_{\theta}}^{h}, 0\right) \cdot \sum_{\theta=1}^{\Theta} v_{q_{\theta}} \Delta^{-1}\left(\tilde{t}_{p q_{\theta}}^{h}, 0\right)\right]
\end{aligned}
$$

Step4: Aggregate the evaluation values given by each expert to obtain the expert group's comprehensive evaluation matrix as shown in formula (9). 


$$
\begin{aligned}
& \tilde{r}_{p q}=\left[\left(r_{p q}, \alpha_{p q}\right),\left(t_{p q}, \varepsilon_{p q}\right)\right] \\
& =\Delta\left[\sum_{h=1}^{H} \lambda_{h} \Delta^{-1}\left(r_{p q}, 0\right) \cdot \sum_{h=1}^{H} \lambda_{h} \Delta^{-1}\left(t_{p q}, 0\right)\right]^{(9)}
\end{aligned}
$$

Step5: Determine PIS $\left(r^{+}\right)$and NIS( $\left.r^{-}\right)$.

$$
\begin{aligned}
& r^{+}=\left(r_{1}^{+}, r_{2}^{+}, \ldots, r_{Q}^{+}\right) \\
& r^{-}=\left(r_{1}^{-}, r_{2}^{-}, \ldots, r_{Q}^{-}\right)
\end{aligned}
$$

Where

$$
r^{+}=\left(r_{q}^{+}, \alpha_{q}^{+}\right)=\left\{\begin{array}{c}
\max _{q}\left\{\left(t_{p q}, \varepsilon_{p q}\right)\right\}, \text { efficiencyindicators } \\
\min _{q}\left\{\left(r_{p q}, \alpha_{p q}\right)\right\}, \text { cost indicators }
\end{array}\right\}
$$

And $r^{-}=\left(r_{q}^{-}, \alpha_{q}^{-}\right)=\left\{\begin{array}{l}\min _{q}\left\{\left(r_{p q}, \alpha_{p q}\right)\right\}, \text { efficiencyindicators } \\ \max _{q}\left\{\left(t_{p q}, \varepsilon_{p q}\right)\right\}, \text { costindicators }\end{array}\right\}$

Step6: Calculate the distance between two interval two-tuple linguistic.

$$
\begin{gathered}
\left(\tilde{d}_{p q}, \alpha_{p q}\right)=\Delta\left(\frac{\Delta^{-1} d\left(r_{q}^{+}, \tilde{r}_{p q}\right)}{\Delta^{-1} d\left(r_{q}^{+}, r_{q}^{-}\right)}\right) \\
\text {Where } d\left(r_{q}^{+}, \tilde{r}_{p q}\right)=\Delta\left[\begin{array}{l}
\frac{1}{2}\left(\begin{array}{l}
\Delta^{-1}\left(r_{q}^{+}, \alpha_{q}^{+}\right)-\Delta^{-1}\left(r_{p q}, \alpha_{p q}\right) \\
+\mid \Delta^{-1}\left(r_{q}^{+}, \alpha_{q}^{+}\right)-\Delta^{-1}\left(t_{p q}, \varepsilon_{p q}\right)
\end{array}\right)
\end{array}\right) \\
d\left(r_{q}^{+}, r_{q}^{-}\right)=\Delta\left(\left|\Delta^{-1}\left(r_{q}^{+}, \alpha_{q}^{+}\right)-\Delta^{-1}\left(r_{q}^{-}, \alpha_{q}^{-}\right)\right|\right)
\end{gathered}
$$

Step7: Calculate positive and negative ideal solutions $d^{+}$ $\left(\tilde{d}_{q}^{+}, \alpha_{q}^{+}\right)$and $d^{-}\left(\tilde{d}_{q}^{-}, \alpha_{q}^{-}\right)$.

Efficiency-type :

$$
d^{+}=\max _{q}\left(\tilde{d}_{p q}, \alpha_{p q}\right), d^{-}=\min _{q}\left(\tilde{d}_{p q}, \alpha_{p q}\right)(17)
$$

Cost-type:

$$
d^{+}=\min _{q}\left(\tilde{d}_{p q}, \alpha_{p q}\right), d^{-}=\max _{q}\left(\tilde{d}_{p q}, \alpha_{p q}\right)
$$

Step8: Calculate the membership degree $\left(h_{p}\right)$. of each new rural cooperative medical institution to the positive ideal solution

$$
h_{p}=\frac{h_{p}^{-}}{h_{p}^{+}+h_{p}^{-}}
$$

Where

$$
h_{p}^{+}=\Delta\left\{\sqrt{\sum_{q=1}^{Q}\left\{\Delta^{-1}\left(w_{q}, \alpha_{q}\right) \cdot\left(\Delta^{-1}\left(\tilde{d}_{p q}, \alpha_{p q}\right)-\Delta^{-1}\left(\tilde{d}_{q}^{+}, \alpha_{q}^{+}\right)\right)\right\}^{2}}\right\}
$$

$$
h_{p}^{-}=\Delta\left\{\sqrt{\sum_{q=1}^{Q}\left\{\Delta^{-1}\left(w_{q}, \alpha_{q}\right) \cdot\left(\Delta^{-1}\left(\tilde{d}_{p q}, \alpha_{p q}\right)-\Delta^{-1}\left(\tilde{d}_{q}^{-}, \alpha_{q}^{-}\right)\right)\right\}^{2}}\right\}
$$

\section{Case Study}

In response to the national related policy, fundamentally solve the problem of rural medical care, at present, many places in China have begun to pilot the new rural cooperative medical care. In order to investigate the quality and effect of the new rural cooperative medical service, we need to evaluate it. One county of S province is one of the national pilot of the policy, we selected four new rural co-operative medical system hospital from this county randomly to evaluate and investigate.In order to effectively carry out this operation, a three-member expert group $\left(D M_{1}\right)$, medical specialists $\left(D M_{2}\right)$, and villagers $\left(D M_{3}\right)$ were established. Three experts used different types of language phrase sets to evaluate, which $D M_{1}$ and $D M_{3}$ using five particle language phrase set $A, D M_{2}$ using seven particle language phrase set $B$. The language phrase set is defined as follows:

$A=\left\{a_{0}: V P(\right.$ very poor $) ; a_{1}: P($ poor $) ; a_{2}: M($ medium $) ; a_{3}:$ $G$ (good); $a_{4}: V G($ very good $\left.)\right\}$;

$B=\left\{b_{0}: V P(\right.$ very poor $) ; b_{1}: P($ poor $) ; b_{2}: M($ medium poor $) ; b_{3}$ : $M($ medium $) ; b_{4}: M G($ medium good $) ; b_{5}: G($ good $) ; b_{6}:$ $V G($ very good $)\} 。$

Three experts on the four new rural cooperative medical care of the original evaluation value shown in Table 2.Because each expert has different knowledge background and social experience, we give $(0.35,0.35,0.30)$ as their weight.. Firstly, the language evaluation information is transformed into the interval two-tuple linguistic, and the first grade index evaluation value is obtained by formula (8). Then the evaluation values of the three experts are gathered by using formula (9) to get the comprehensive evaluation value of expert group as shown in Table 3. And $r^{+}=\{\Delta(0.811), \quad \Delta(0.757), \quad \Delta(0.748), \quad \Delta(0.785), \quad \Delta(0.596)$, $\Delta(0.831)\}, r^{-}=\{\Delta(0.615), \quad \Delta(0.563), \quad \Delta(0.617), \quad \Delta(0.526)$, $\Delta(0.791), \Delta(0.581)\}$ are obtained by the formula (10-13). Then $d^{+}=\{\Delta(0.675), \Delta(0.598), \Delta(0.752), \Delta(0.640), \Delta(0.352)$, $\Delta(0.829)\}, \quad d=\{\Delta(0.193), \Delta(0.284), \Delta(0.144), \Delta(0.093)$, $\Delta(0.752), \Delta(0.325)\}$ were calculated by using TOPSIS method. Then, four new rural cooperative medical institutions were sorted according to the comprehensive evaluation value. Finally, the final evaluation results are shown in Table 4.The results show that the service quality of the third NCMS is the best compared to the other three, and the fourth NCMS is the worst

Table 2: New Rural Cooperative Medical Service Original 
International Journal of Science and Research (IJSR)

ISSN (Online): 2319-7064

Index Copernicus Value (2015): 78.96 | Impact Factor (2015): 6.391

\begin{tabular}{|l|c|c|c|c|c|c|c|c|c|c|c|c|}
\hline & \multicolumn{10}{|c|}{$D M_{1}$} & \multicolumn{3}{c|}{$D M_{2}$} & \multicolumn{4}{|c|}{$D M_{3}$} \\
\hline & $A_{1}$ & $A_{2}$ & $A_{3}$ & $A_{4}$ & $A_{1}$ & $A_{2}$ & $A_{3}$ & $A_{4}$ & $A_{1}$ & $A_{2}$ & $A_{3}$ & $A_{4}$ \\
\hline$C_{11}$ & $\mathrm{G}$ & $\mathrm{G}-\mathrm{VG}$ & $\mathrm{M}$ & $\mathrm{M}-\mathrm{G}$ & $\mathrm{M}$ & $\mathrm{MG}$ & $\mathrm{M}-\mathrm{G}$ & $\mathrm{MP}-\mathrm{G}$ & $\mathrm{G}$ & $\mathrm{M}-\mathrm{G}$ & $\mathrm{VG}$ & $\mathrm{G}$ \\
\hline$C_{12}$ & $\mathrm{VG}$ & $\mathrm{G}-\mathrm{VG}$ & $\mathrm{G}$ & $\mathrm{M}$ & $\mathrm{G}-\mathrm{VG}$ & $\mathrm{VG}$ & $\mathrm{M}-\mathrm{G}$ & $\mathrm{G}$ & $\mathrm{M}-\mathrm{G}$ & $\mathrm{VG}$ & $\mathrm{M}$ & $\mathrm{G}$ \\
\hline$C_{13}$ & $\mathrm{M}$ & $\mathrm{G}$ & $\mathrm{P}-\mathrm{G}$ & $\mathrm{G}$ & $\mathrm{M}-\mathrm{G}$ & $\mathrm{MG}$ & $\mathrm{G}$ & $\mathrm{M}-\mathrm{G}-$ & $\mathrm{VG}$ & $\mathrm{M}$ & $\mathrm{M}-\mathrm{VG}$ & $\mathrm{M}-\mathrm{G}$ \\
\hline$C_{14}$ & $\mathrm{G}$ & $\mathrm{M}$ & $\mathrm{VG}$ & $\mathrm{M}$ & $\mathrm{MG}$ & $\mathrm{G}-\mathrm{VG}$ & $\mathrm{M}$ & $\mathrm{G}$ & $\mathrm{G}$ & $\mathrm{VG}$ & $\mathrm{M}-\mathrm{G}$ & $\mathrm{G}$ \\
\hline$C_{21}$ & $\mathrm{VG}$ & $\mathrm{G}-\mathrm{VG}$ & $\mathrm{M}$ & $\mathrm{G}$ & $\mathrm{MP}$ & $\mathrm{M}-\mathrm{G}$ & $\mathrm{G}$ & $\mathrm{MP}-\mathrm{M}$ & $\mathrm{G}$ & $\mathrm{M}$ & $\mathrm{M}-\mathrm{VG}$ & $\mathrm{M}$ \\
\hline$C_{22}$ & $\mathrm{G}$ & $\mathrm{M}$ & $\mathrm{M}-\mathrm{G}$ & $\mathrm{M}-\mathrm{G}$ & $\mathrm{G}$ & $\mathrm{MP}-\mathrm{G}$ & $\mathrm{MG}$ & $\mathrm{VG}$ & $\mathrm{M}$ & $\mathrm{G}$ & $\mathrm{VG}$ & $\mathrm{G}$ \\
\hline$C_{23}$ & $\mathrm{M}-\mathrm{G}$ & $\mathrm{G}$ & $\mathrm{M}$ & $\mathrm{P}-\mathrm{G}$ & $\mathrm{MP}$ & $\mathrm{M}-\mathrm{G}$ & $\mathrm{G}$ & $\mathrm{MG}$ & $\mathrm{G}$ & $\mathrm{M}$ & $\mathrm{M}-\mathrm{G}$ & $\mathrm{M}$ \\
\hline$C_{31}$ & $\mathrm{VG}$ & $\mathrm{M}$ & $\mathrm{M}-\mathrm{G}$ & $\mathrm{M}$ & $\mathrm{MG}$ & $\mathrm{MP}-\mathrm{M}$ & $\mathrm{G}$ & $\mathrm{M}-\mathrm{G}$ & $\mathrm{G}$ & $\mathrm{G}$ & $\mathrm{M}-\mathrm{G}$ & $\mathrm{M}-\mathrm{G}$ \\
\hline$C_{32}$ & $\mathrm{G}$ & $\mathrm{M}-\mathrm{G}$ & $\mathrm{M}$ & $\mathrm{G}$ & $\mathrm{G}$ & $\mathrm{MG}$ & $\mathrm{M}-\mathrm{VG}$ & $\mathrm{M}-\mathrm{G}$ & $\mathrm{M}-\mathrm{G}$ & $\mathrm{M}$ & $\mathrm{VG}$ & $\mathrm{G}$ \\
\hline$C_{33}$ & $\mathrm{M}-\mathrm{G}$ & $\mathrm{VG}$ & $\mathrm{G}$ & $\mathrm{M}$ & $\mathrm{MG}$ & $\mathrm{G}$ & $\mathrm{MP}-\mathrm{M}$ & $\mathrm{MG}$ & $\mathrm{G}$ & $\mathrm{M}$ & $\mathrm{G}$ & $\mathrm{M}-\mathrm{VG}$ \\
\hline$C_{34}$ & $\mathrm{M}$ & $\mathrm{M}-\mathrm{G}$ & $\mathrm{VG}$ & $\mathrm{G}$ & $\mathrm{VG}$ & $\mathrm{M}-\mathrm{VG}$ & $\mathrm{MG}$ & $\mathrm{G}$ & $\mathrm{M}$ & $\mathrm{M}-\mathrm{G}$ & $\mathrm{M}$ & $\mathrm{G}$ \\
\hline$C_{41}$ & $\mathrm{G}$ & $\mathrm{G}$ & $\mathrm{M}-\mathrm{G}$ & $\mathrm{M}$ & $\mathrm{MP}-\mathrm{G}$ & $\mathrm{MG}$ & $\mathrm{G}$ & $\mathrm{M}-\mathrm{G}$ & $\mathrm{M}-\mathrm{G}$ & $\mathrm{M}$ & $\mathrm{G}$ & $\mathrm{M}-\mathrm{VG}$ \\
\hline$C_{42}$ & $\mathrm{G}$ & $\mathrm{M}$ & $\mathrm{VG}$ & $\mathrm{M}-\mathrm{G}$ & $\mathrm{M}-\mathrm{G}$ & $\mathrm{MG}$ & $\mathrm{G}$ & $\mathrm{MG}$ & $\mathrm{M}$ & $\mathrm{G}$ & $\mathrm{M}$ & $\mathrm{M}$ \\
\hline$C_{51}$ & $\mathrm{P}-\mathrm{VG}$ & $\mathrm{M}-\mathrm{G}$ & $\mathrm{G}$ & $\mathrm{M}$ & $\mathrm{MG}$ & $\mathrm{VG}$ & $\mathrm{M}-\mathrm{G}$ & $\mathrm{MG}$ & $\mathrm{M}$ & $\mathrm{G}$ & $\mathrm{M}-\mathrm{G}$ & $\mathrm{M}-\mathrm{G}$ \\
\hline$C_{52}$ & $\mathrm{VG}$ & $\mathrm{M}-\mathrm{G}$ & $\mathrm{G}$ & $\mathrm{M}$ & $\mathrm{G}$ & $\mathrm{M}-\mathrm{G}$ & $\mathrm{M}$ & $\mathrm{VG}$ & $\mathrm{G}$ & $\mathrm{M}-\mathrm{G}$ & $\mathrm{M}$ & $\mathrm{G}$ \\
\hline$C_{53}$ & $\mathrm{G}$ & $\mathrm{M}$ & $\mathrm{M}-\mathrm{G}$ & $\mathrm{G}$ & $\mathrm{VG}$ & $\mathrm{M}-\mathrm{VG}$ & $\mathrm{G}$ & $\mathrm{MG}$ & $\mathrm{M}$ & $\mathrm{G}$ & $\mathrm{M}-\mathrm{VG}$ & $\mathrm{M}$ \\
\hline$C_{61}$ & $\mathrm{VG}$ & $\mathrm{G}$ & $\mathrm{M}$ & $\mathrm{M}-\mathrm{G}$ & $\mathrm{M}$ & $\mathrm{M}-\mathrm{G}$ & $\mathrm{M}-\mathrm{VG}$ & $\mathrm{G}$ & $\mathrm{G}$ & $\mathrm{M}$ & $\mathrm{G}-\mathrm{VG}$ & $\mathrm{M}$ \\
\hline$C_{62}$ & $\mathrm{M}$ & $\mathrm{M}-\mathrm{G}$ & $\mathrm{G}$ & $\mathrm{M}$ & $\mathrm{MG}$ & $\mathrm{M}-\mathrm{G}$ & $\mathrm{VG}$ & $\mathrm{MG}-\mathrm{G}$ & $\mathrm{M}-\mathrm{G}$ & $\mathrm{G}$ & $\mathrm{M}-\mathrm{G}$ & $\mathrm{M}$ \\
\hline
\end{tabular}

Table 3: Expert Group Integrated Evaluation Value

\begin{tabular}{|c|c|c|c|c|c|c|}
\hline & $\mathrm{C} 1$ & $C_{2}$ & $C_{3}$ & $C_{4}$ & $C_{5}$ & $C_{6}$ \\
\hline \multirow{4}{*}{$A_{1}$} & $\Delta[0.703$, & $\Delta[0.644$, & $\Delta[0.710$, & $\Delta[0.555$, & $\Delta[0.697$, & $\Delta[0.654$, \\
& $0.759]$ & $0.670]$ & $0.747]$ & $0.745]$ & $0.789]$ & $0.692]$ \\
\hline \multirow{2}{*}{$A_{2}$} & $\Delta[0.735$, & $\Delta[0.563$, & $\Delta[0.590$, & $\Delta[0.640$, & $\Delta[0.610$, & $\Delta[0.581$, \\
& $0.789]$ & $0.757]$ & $0.709]$ & $0.640]$ & $0.791]$ & $0.742]$ \\
\hline \multirow{2}{*}{$A_{3}$} & $\Delta[0.615$, & $\Delta[0.649$, & $\Delta[0.649$, & $\Delta[0.737$, & $\Delta[0.596$, & $\Delta[0.669$, \\
& $0.771]$ & $0.754]$ & $0.748]$ & $0.785]$ & $0.761]$ & $0.831]$ \\
\hline \multirow{2}{*}{$A_{4}$} & $\Delta[0.622$, & $\Delta[0.589$, & $\Delta[0.617$, & $\Delta[0.526$, & $\Delta[0.652$, & $\Delta[0.588$, \\
& $0.735]$ & $0.693]$ & $0.735]$ & $0.712]$ & $0.678]$ & $0.660]$ \\
\hline
\end{tabular}

Table 4: Final Evaluation Results

\begin{tabular}{|l|l|l|l|l|}
\hline & $A_{1}$ & $A_{2}$ & $A_{3}$ & $A_{4}$ \\
\hline$C_{1}$ & $\Delta(0.410)$ & $\Delta(0.193)$ & $\Delta(0.602)$ & $\Delta(0.675)$ \\
\hline$C_{2}$ & $\Delta(0.516)$ & $\Delta(0.500)$ & $\Delta(0.284)$ & $\Delta(0.598)$ \\
\hline$C_{3}$ & $\Delta(0.144)$ & $\Delta(0.752)$ & $\Delta(0.376)$ & $\Delta(0.548)$ \\
\hline$C_{4}$ & $\Delta(0.520)$ & $\Delta(0.559)$ & $\Delta(0.093)$ & $\Delta(0.640)$ \\
\hline$C_{5}$ & $\Delta(0.753)$ & $\Delta(0.535)$ & $\Delta(0.423)$ & $\Delta(0.352)$ \\
\hline$C_{6}$ & $\Delta(0.633)$ & $\Delta(0.679)$ & $\Delta(0.325)$ & $\Delta(0.829)$ \\
\hline$h_{p}$ & $\Delta(0.411)$ & $\Delta(0.767)$ & $\Delta(0.315)$ & $\Delta(0.955)$ \\
\hline & $\left(a_{2},-0.099\right)$ & $\left(a_{3}, 0.017\right)$ & $\left(a_{1}, 0.065\right)$ & $\left(a_{4},-0.045\right)$ \\
\hline
\end{tabular}

\section{Conclusion}

In this paper, a new method of multi-attribute group decision-making based on interval two-tuple linguistic for the evaluation of new rural cooperative medical service is proposed in order to solve the problem of rural medical care.In the multi-attribute decision-making problem, both of the decision-making process and the decision-making problem itself are uncertain, so we use the language phrases evaluation set to evaluate the medical service. In order to avoid the loss of evaluation information in the process of aggregating linguistic information, this paper uses interval two-tuple linguistic aggregation operator to aggregate the evaluation information. Based on the existing research, this paper constructs a new rural cooperative medical service evaluation index system by generalizing and summarizing the evaluation index of medical service and combining the expert interview and questionnaire survey.New rural cooperative medical system is not only the promotion of national policy, but also to better meet the needs of local villagers, therefore, this expert group in addition to medical experts, also joined the local villagers and government. Finally, the TOPSIS method is used to solve the problem

\section{References}

[1] Li X.D. Research on the Current Situation and Development of New Rural Cooperative Medical System [D]. Master 's Degree Thesis of Jiangsu University, 2010.

[2] Zhang G.K. The Present Situation and Prospects of the New Rural Cooperative Medical[J].Agricultural Economy, 2010, (1):67-73.

[3] Hu S.L. Analysis on the Operation and Evaluation of New Rural Cooperative Medical System in China [J]. Chinese Health Economics, 2008, 27(2):26-27.

[4] Meng H.B, Wang Z.B. Research on the Financing Mechanism of New Rural Cooperative Medical System and the Game of Stakeholders [J]. Journal of China Agricultural University, 2007, 24(1):122-129.

[5] Wang J.H. Research on Supply and Practical Demand of New Rural Cooperative Medical System in China [J]. China Soft Science, 2007, (1):10-14.

[6] Peng J, Jiang Q.C, Wang H.P. An Analysis on the Operation of Out - patient Co - ordination System of New Rural Cooperative Medical System in Anhui Province $[\mathrm{J}]$. Chinese Health Service Management, 2009, 26(6):400-400.

[7] Herrera F, Martínez L, Herrera F. A 2-tuple fuzzy linguistic representation model for computing with words[J]. Fuzzy Systems IEEE Transactions on, 2000, 8(6):746 - 752 .

[8] Tai W, Chen C. A new evaluation model for 


\section{International Journal of Science and Research (IJSR) \\ ISSN (Online): 2319-7064}

Index Copernicus Value (2015): 78.96 | Impact Factor (2015): 6.391

intellectual capital based on computing with linguistic variable[J]. Expert Systems with Applications An International Journal, 2009, 36(2):3483-3488.

[9] You X, You J, Liu H, et al. Group multi-criteria supplier selection using an extended VIKOR method with interval 2-tuple linguistic information[J]. Expert Systems with Applications, 2015, 42:1906-1916.

[10] $\mathrm{H}$ Zhang. The multi-attribute group decision making method based on aggregation operators with interval-valued 2-tuple linguistic information[J]. Mathematical \& Computer Modelling, 2012, 56:27-35.

[11] Yildlz Z, Erdoğmuş S. Measuring Patient Satisfaction of the Quality of Health Care: A Study of Hospitals in Turkey[J]. Journal of Medical Systems, 2004, 28(6):581-589.

[12] Shieh J, Wu H, Huang K. A DEMATEL method in identifying key success factors of hospital service quality[J]. Knowledge-Based Systems, 2010, 23(3):277-282.

[13] Lynn M R, McMillen B J, Sidani S. Understanding and measuring patients' assessment of the quality of nursing care.[J]. Nursing Research, 2007, 56(3):159-166.

[14] Chang T H. Fuzzy VIKOR method: A case study of the hospital service evaluation in Taiwan[J]. Information Sciences, 2014, 271(7):196-212 